\title{
Using a Stochastic Model to Detect Unusual Continuous Glucose Monitor Behaviour in Newborn Infants
}

\author{
Matthew SIGNAL ${ }^{1}$, Aaron LE COMPTE ${ }^{1}$, Deborah L HARRIS ${ }^{2,3}$, \\ Phil J WESTON ${ }^{2}$, Jane E HARDING ${ }^{3}$, J. Geoffrey CHASE ${ }^{1}$ on behalf of the CHYLD Study Group
}

\author{
1. Department of Mechanical Engineering, University of Canterbury, Christchurch, New Zealand \\ 2. Newborn Intensive Care Unit, Waikato District Health Board, New Zealand \\ 3. Liggins Institute, University of Auckland, Auckland, New Zealand
}

\begin{abstract}
Abnormal blood glucose (BG) concentrations have been associated with negative outcomes in critically ill adults and infants. Diagnosis of hyperglycaemia and hypoglycaemia is by BG measurements, which are typically taken several hours apart due to the clinical effort required. Continuous glucose monitoring (CGM) devices, which take measurements every 5 minutes, have the potential to improve the detection and diagnosis of these glycaemic abnormalities. There have been relatively few successful investigations of CGM devices in the ICU, and one study reported significant sensor noise. If CGM devices are going to be used in the clinical setting to monitor, diagnose and potentially treat critical illness, clinicians need to know data are reliable and accurate. This study uses CGM data from neonatal infants to develop a tool that will aid clinicians in identifying unusual CGM behaviour. A stochastic model was created to classify CGM measurements with the aim of highlighting unusual CGM behaviour. In addition, the method uses a colour coded CGM trace to convey the information quickly and efficiently, either retrospectively or in real-time. The method has been used to detect unusual hypoglycaemic events and potential sensor degradation, both of which need to be interpreted with care. Overall, while BG measurements are required to make definitive conclusions about glycaemic events, the stochastic model provides another level of information to aid users in interpretation and decision making.
\end{abstract}

Keywords: hypoglycaemia, continuous glucose monitoring, modelling, detection, critically ill, infant, preterm

\section{INTRODUCTION}

Abnormal blood glucose (BG) concentrations have been associated with increased morbidity and mortality in both critically ill adults and infants. Patients in the intensive care unit (ICU) often experience high levels of insulin resistance and stress-induced hyperglycaemia, which can negatively impact outcomes (Capes et al., 2000, Finney et al., 2003, Krinsley, 2003, Bistrian, 2001, Van den Berghe et al., 2001). Further complicating the matter, hypoglycaemia and glycaemic variability have both been independently linked to mortality in critically ill patients (Egi et al., 2006, Egi et al., 2010, Hermanides et al., 2010, Krinsley, 2008).

Diagnosis of hyperglycaemia and hypoglycaemia in critically ill patients is by blood glucose (BG) measurements, which are typically taken several hours apart. More frequent BG measurements are not clinically practical due to the additional nursing workload (Carayon and Gurses, 2005, Chase et al., 2008, Mackenzie et al., 2005) and consequently, important glycaemic events between BG measurements can go undetected. Continuous Glucose Monitoring (CGM) devices have the potential to improve the detection and diagnosis of these glycaemic abnormalities. The continuous glucose monitoring system (CGMS ${ }^{\circledR}$ System Gold ${ }^{\mathrm{TM}}$ Medtronic, Minimed, Northridge, CA, USA) provides a glucose value every 5 minutes or 288 measurements per day, with only 4 BG measurements per day required for device calibration.

There have been relatively few successful investigations of CGMs in critical care use (Goldberg et al., 2004), although they are well studied in Type 1 diabetes (Breton and Kovatchev, 2008, Klonoff, 2005a, Klonoff, 2005b). In particular, one set of tight glycaemic control trials using CGM technology was not particularly successful due, in part, to significant sensor noise (Chee et al., 2003a, Chee et al., 2003b). Added sensor noise is a trade off (in some cases) for the CGMs far higher, automated sampling rate (Goldberg et al., 2004, Clarke et al., 2005) and must be effectively managed for these devices to be used successfully. However, these sensor and algorithm technologies are also constantly evolving with every new generation offering improvements (Klonoff, 2005b, Skyler, 2009).

If CGM devices are going to be used in the clinical setting to monitor, diagnose and potentially treat critical illness, clinicians need to know the data is reliable and accurate. Consider a scenario in which CGM data are retrospectively analysed to classify hypoglycaemia in neonates, where frequent BG measurements are not available. Three consecutive measurements in a CGM trace read $4 \mathrm{mmol} / \mathrm{L}$, $2.5 \mathrm{mmol} / \mathrm{L}$, followed by $4 \mathrm{mmol} / \mathrm{L}$. If hypoglycaemia was classified as a measurement below $2.6 \mathrm{mmol} / \mathrm{L}$, then this 
would be recorded as a hypoglycaemic event. However, if the rest of the CGM trace was very stable with low variability, intuition would suggest this 'event' is potentially a sensor artefact.

Our manuscript describes a tool that will aid clinicians in identifying unusual CGM behaviour, retrospectively or in real-time, and highlight sections of the CGM glucose trace that potentially need to be interpreted with care.

\section{SUBJECTS AND METHODS}

\subsection{Subjects}

This study uses CGM data from 50 babies at risk of hypoglycaemia who were admitted to the Waikato Hospital Newborn Intensive Care Unit (NICU). Table 1 presents the cohort demographics for the 50 patients. Demographics are presented as median [interquartile range] where applicable.

Table 1: Patient demographics

\begin{tabular}{|l|c|}
\hline Cohort Demographics & \\
\hline Number of CGM traces & 50 \\
\hline Sex (M/F) & $26 / 24$ \\
\hline Gestational Age (weeks) & $34[33-37]$ \\
\hline Birthweight (g) & $2172[1880-2990]$ \\
\hline Primary Risk (\# infants): & 15 \\
\hline Diabetes & 19 \\
\hline Premature & 14 \\
\hline Small or Large for gestational age & 2 \\
\hline Other & \\
\hline
\end{tabular}

\subsection{Continuous Glucose Monitoring}

All patients had interstitial glucose monitoring using the CGMS ${ }^{\circledR}$ System Gold ${ }^{\mathrm{TM}}$. Monitoring began on admission to the NICU and finished after 7 days or earlier if the baby was no longer considered at risk of hypoglycaemia. During the monitoring period nurses were asked to record all blood glucose concentrations, feeding and medication for the management of hypoglycaemia. However, they remained blind to the glucose concentrations determined by the device. The device was calibrated as per the manufacturer's recommendations and all of the data entered into the device were checked against clinical records for accuracy. Upon completion of continuous glucose monitoring, data were downloaded to a PC using CGMS system solutions software version $3.0 \mathrm{C}$, which calibrated the CGM readings retrospectively.

\subsection{Calibration Measurements}

All BG calibration measurements were determined by a blood gas analyser (Radiometer, ABL800Flex, Copenhagen) using the glucose oxidase method. This device has a reading range of 0.0 to $60.0 \mathrm{mmol} / \mathrm{L}$ and a coefficient of variation of $2.1 \%$ (Harris et al., 2010). Due to the location of the blood gas analyser, a short time delay (estimated $<25 \mathrm{mins}$ maximum) was possible between taking the blood sample and entering the calibration glucose level into the device.

\subsection{Stochastic Model and CGM Classification}

A stochastic model based on the kernel density method was used to classify CGM measurements as expected or unexpected, using the previous CGM measurement and information about the history of CGM behaviour. The model is an extension to the methods described by Lin et al (Lin et al., 2008) who developed a stochastic model for insulin sensitivity prediction.

The CGM data sets are broken down into paired, consecutive CGM measurements $\left(C G M_{n-1}, C G M_{n}\right)$. Kernel density estimation methods are used to create a smooth, continuous, non-parametric model surface that reflects the data pattern. For every possible $C G M_{n-1}$, the stochastic model provides a continuous, empirical estimate of the conditional probability density function (pdf) for the next CGM measurement, $C G M_{n}$. These conditional pdf's provide the basis for classifying CGM measurements and identifying unusual CGM behaviour.

Using the stochastic model, a given CGM measurement, $C G M_{n}$, would be classified as follows:

1. The previous measurement, $C G M_{n-1}$, is used to find the corresponding conditional pdf from the model.

2. $C G M_{n}$ is located in the pdf and its percentile value in the conditional pdf is determined.

3. The percentile is used to classify $C G M_{n}$, where a very high or very low percentile is indicative of an outlier. These outliers are classified as unusual CGM measurements.

The measurement-to-measurement sections of the CGM trace were colour coded based on the percentile value, to highlight areas of unusual CGM behaviour quickly and effectively. Three confidence intervals (CI's) were used to specify the colour: within $80 \%$ CI $\left(10^{\text {th }}-90^{\text {th }}\right.$ percentile $)$ was blue, within $90 \%$ CI $\left(5^{\text {th }}-95^{\text {th }}\right.$ percentile $)$ was cyan, within $99 \%$ CI $\left(0.5^{\text {th }}\right.$ $99.5^{\text {th }}$ percentile) was yellow, and outside $99 \%$ CI was red. These intervals were chosen based on the data used in this study and can be customised for different patient groups and/or different CGM sensors. As the scale starts at $80 \% \mathrm{CI}$, the focus here is on classifying outliers, rather than the full range.

\section{RESULTS}

Figure 1 shows a plot all of the CGM data $\left(C G M_{n-1}, C G M_{n}\right)$. The contour lines represent the 5th, 25th, 50th, 75th, and 95th percentiles of the stochastic model surface. Figure 2 shows a distribution of the data density by glycaemic level. Figure 3 shows a surface plot of the stochastic model. Conditional probability density functions are slices parallel to the $C G M_{n}$ axis, and each slice has an area under the curve of 1.0. Figure 4 shows a comparison of the pdf's obtained from the model versus the pdf's obtained directly from the CGM data. Each pdf shows the expected distribution of $C G M_{n}$ given a 
previous measurement $\left(C G M_{n-1}\right)$ of $2,4,6,8$ or $10 \mathrm{mmol} / \mathrm{L}$. It should be noted that the pdf's could be generated for any value of $C G M_{n-1}$ within the bounds of the model; Figure 4 shows just five examples.

Figures 5-7 show three examples of CGM traces that have been coloured using the stochastic classification method. Figure 5 shows a stable trace, which is almost entirely dark blue, indicating the measurement-to-measurement change

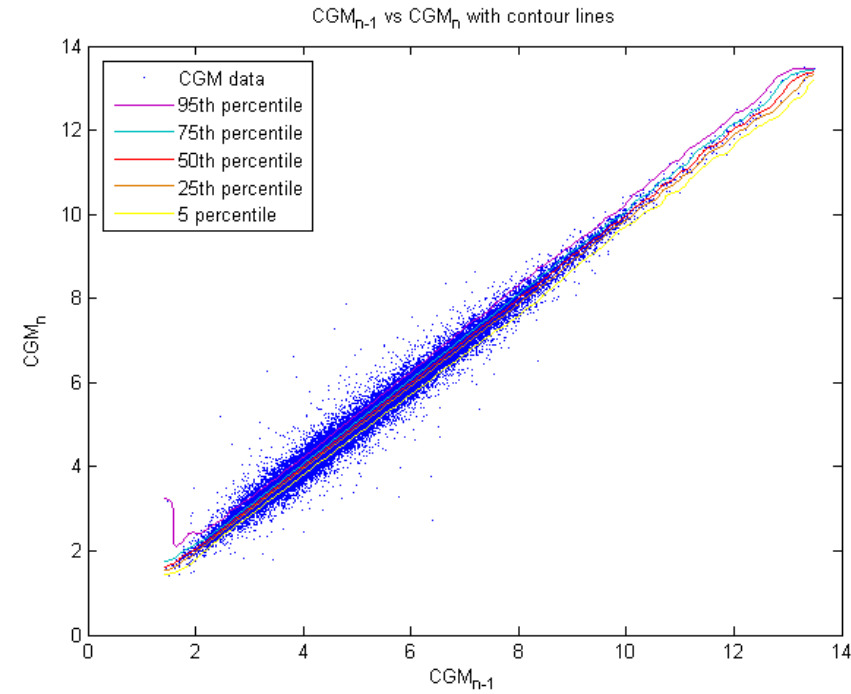

Figure 1: Plot of CGM measurement pairs $\left(C G M_{n-1}, C G M_{n}\right)$ with contour lines representing the $5^{\text {th }}$ to $95^{\text {th }}$ percentiles, from the bottom of the plot up.

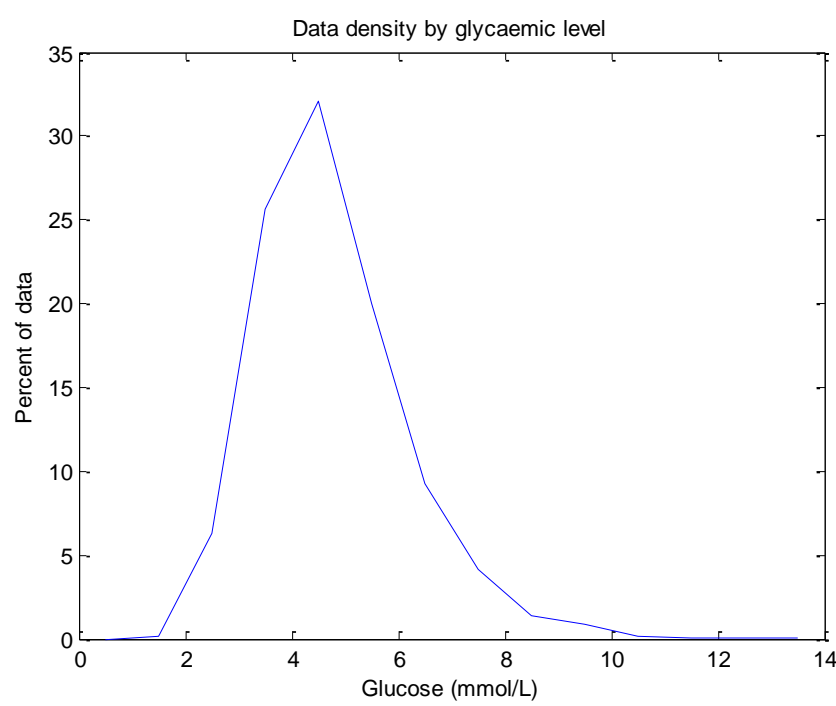

Figure 2: Density of the data set by glycaemic level. Density is shown as a percent of the total data set $(67,438$ measurements). throughout the trace is not unusual. Figure 6 shows a trace with several potentially unusual measurements throughout the trace. The hypoglycaemic event that occurs at approximately one day after monitoring began is coloured red and classified as very unusual. Figure 7 shows a trace with a few potentially unusual measurements for the first three days of monitoring. After day 3 a high proportion of the CGM measurements are classified as very unusual and are coloured red.

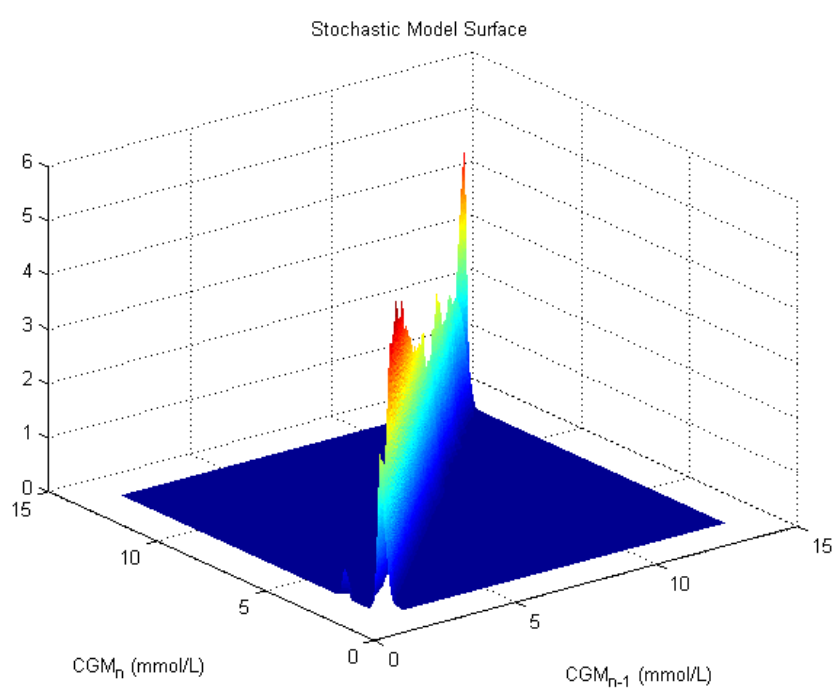

Figure 3: Stochastic model surface for this data set

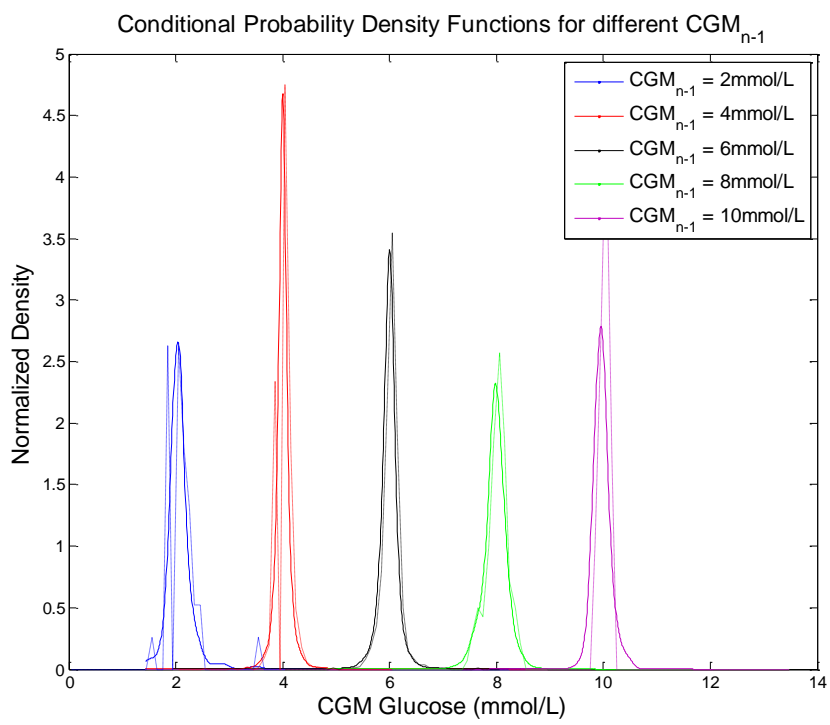

Figure 4: Comparison of conditional probability density functions at different $C G M_{n-1}$. Pdf's from the model are solid lines and empirical pdf's from actual CGM data are dotted 


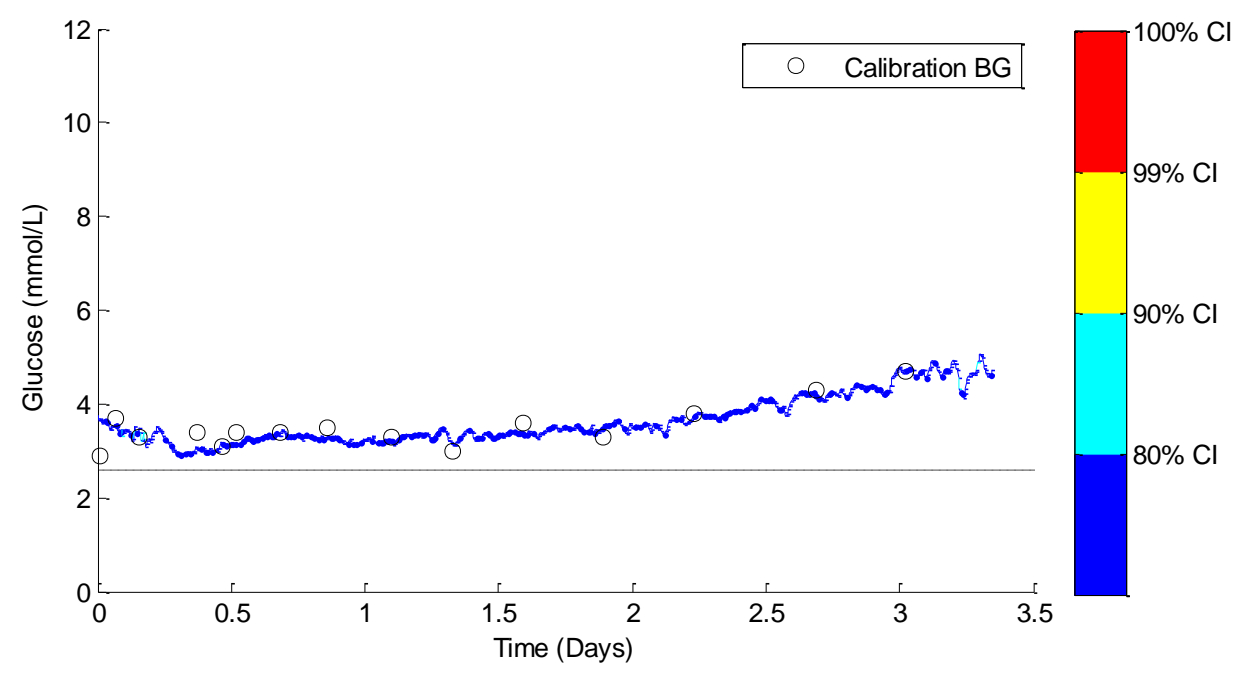

Figure 5: Stable CGM trace with no yellow or red measurements indicating no CGM measurements were classified unusual.

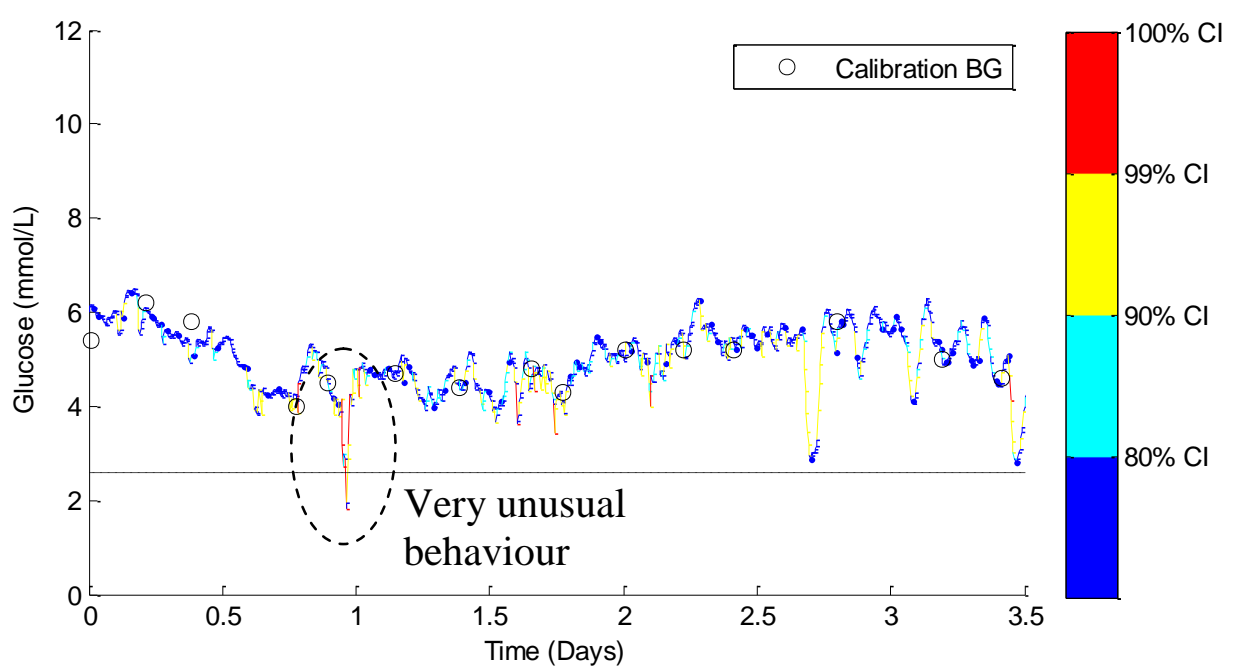

Figure 6: CGM trace with several measurements classified as mildly unusual. Note the hypoglycaemic event at $\sim 1$ day which has been classified as very unusual (red).

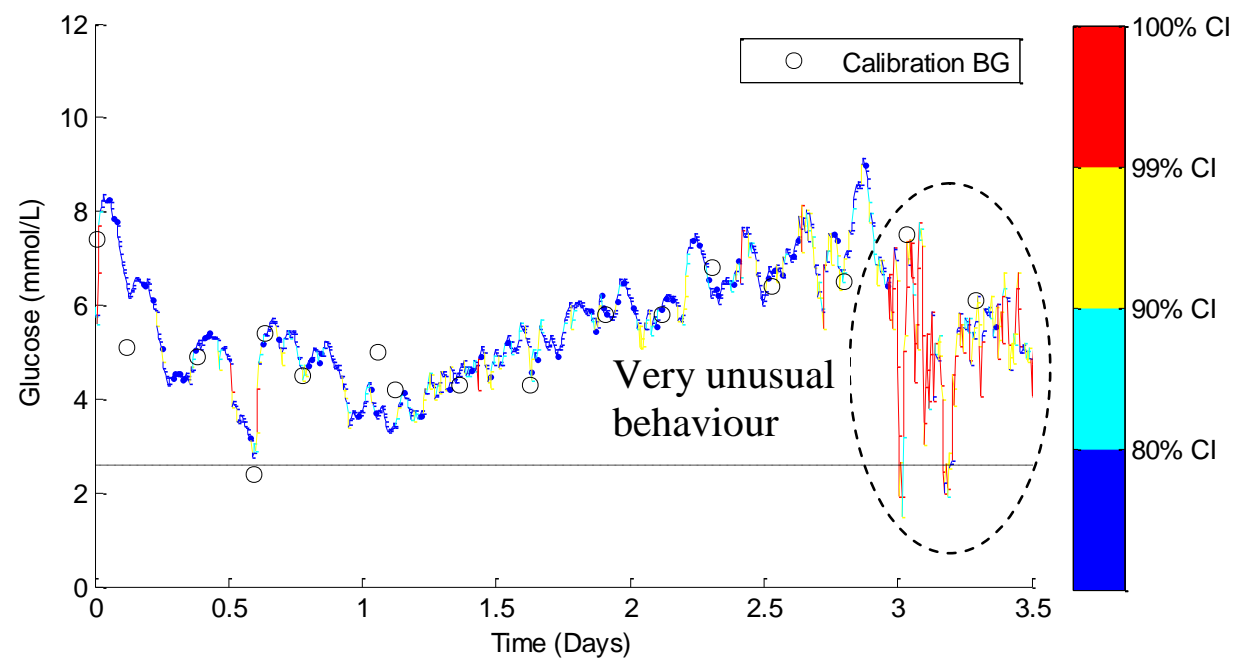

Figure 7: CGM trace with several measurements classified as mildly unusual. After day 3 the trace is classified as very unusual (red) and could be indicative of sensor malfunction. 


\section{DISCUSSION}

The aim of this study was to design a tool that could aid clinicians in identifying unusual CGM behaviour that should potentially be interpreted with care. Stochastic modelling methods from (Lin et al., 2008) and a method of colouring CGM traces were used to highlight unusual CGM behaviour clearly and efficiently, in either real-time or retrospectively.

Figures 1 and 2 give information about the raw data used to create the stochastic model. More than $99 \%$ of the data is within $2-10 \mathrm{mmol} / \mathrm{L}$ range, shown in Figure 2. There are several outliers in Figure 1 that have a very large change in glycaemia over the 5 minute measurement interval. The high data density means these outliers have little effect on the model fit, shown by the smooth and tight percentile lines in Figure 1. However, below $2 \mathrm{mmol} / \mathrm{L}$ there are $97 \mathrm{CGM}$ measurements and due to the relatively low density of data the outliers have more impact on the model fit. This effect is shown by the 95th percentile line of the model, which strays upward at levels below 2mmol/L. Similarly, above $10 \mathrm{mmol} / \mathrm{L}$ there are only 232 measurements and the percentile lines all have a wave-like shape, again showing the effect of outliers where data density is low. A greater data density would alleviate these issues without changing the approach in this proof-of-concept.

Figure 3 shows the surface of the stochastic model. The colour gradient shows how the shape of the model changes in the domain of $C G M_{n-1}$ and that a single, global probability density function is not applicable to this data set. Figure 4 further reinforces this with 5 pdf's taken from the model at different $C G M_{n-1}$ values, resulting in 5 different shaped density functions. These pdf's are also used to show that the model fits the empirical data well. The model pdf's (solid lines) overlay the empirical data (dotted lines) with only minor discrepancies.

Figures 5-7 show 3 different CGM data sets and how the stochastic model classified the individual CGM measurements within them. Figure 5 shows a very stable, flat CGM trace with only small variations over the 3.5 days of monitoring. The CGM trace passes near all calibration measurements and there doesn't appear to be any unusual CGM behaviour. The stochastic model classified almost the entire trace as dark blue indicating no unusual CGM behaviour. The interpretation of this trace would not likely be influenced with the additional information provided by the model.

Figure 6 shows a less stable CGM trace with a lot more variability. This trace contains a few yellow and red sections that potentially need to be interpreted with care. The focus of this discussion is the hypoglycaemic event that occurs at $\sim$ day 1 . In the sequence of 5 measurements that lead up to the $1.8 \mathrm{mmol} / \mathrm{L}$ minimum, there are two drops of $\sim 1 \mathrm{mmol} / \mathrm{L}$ per 5 minute measurement interval. The model has determined these are extreme outliers and consequently they have been coloured red. The trace then rises to above $4 \mathrm{mmol} / \mathrm{L}$ in 5 measurements, similarly with two rises of $\sim 1 \mathrm{mmol} / \mathrm{L}$ per 5 minute measurement interval. Although the physiological limits of glucose rate-of-change are still unknown, the level of sensor error that has been reported in previous CGM studies (Breton and Kovatchev, 2008, Goldberg et al., 2004) suggest that this hypoglycaemic event could potentially be either glycaemia or sensor error.

It is important to note that the aim of the stochastic model presented is not to try and determine the cause of the drop in CGM glucose, but rather to highlight the fact it should be interpreted with care. Furthermore, if the stochastic model was implemented in a real-time clinical setting and the downward CGM measurements were observed, it would be beneficial for the clinician to know whether the sequence of measurements is typical of CGM devices and that patient cohort. It should also be noted that without an accurate BG measurement at $\sim 1$ day, no exact conclusion can be drawn about the whether the hypoglycaemic event in this data was due to sensor error, or a true glycaemic event.

However, this lack of confirmation is often the reality with CGMs. Clinical protocols might use stochastic information to justify an added calibration measurement to clarify a potentially significant event. After an event, such traces would yield insight not present at the bedside.

Figure 7 shows an example of CGM data that becomes increasingly more variable and unstable at approximately day 3 of monitoring. Before day 3, the CGM trace is predominantly blue and cyan with only small patches of yellow and occasionally red. However, after day the 3 the CGM trace is almost entirely red indicating the stochastic model has classified these measurements as very unusual. The sudden apparent degradation of reliable CGM measurements could be due to a sensor failure. This is not an unreasonable hypothesis, given the sensors used in this study were validated for 3 days of continuous monitoring. Again, without more frequent, accurate BG measurements during the period after day 3 no definitive conclusions can be drawn. However, this example represents another potential use of this stochastic model classification method that might be useful to users of CGM devices.

\section{CONCLUSIONS}

This study has produced a stochastic model capable of classifying CGM measurements with the aim of highlighting unusual CGM behaviour. The method uses a colour coded CGM trace to convey the information quickly and efficiently and it is computationally light enough to be used retrospectively or in real-time.

There are several potential uses for the stochastic classification which include, but are not limited to, classification of hypoglycaemia and detection of potential sensor failure. Equally, they can augment alarming methods or be used to more optimally time BG measurements in cohorts, such as neonates, where blood draws are restricted. Overall, while BG measurements are required to make definitive conclusions about glycaemic events, the stochastic model provides another level of information to aid users in interpretation and decision making. 


\section{REFERENCES}

BISTRIAN, B. R. 2001. Hyperglycemia and infection: which is the chicken and which is the egg? JPEN $J$ Parenter Enteral Nutr, 25, 180-181.

BRETON, M. \& KOVATCHEV, B. 2008. Analysis, modeling, and simulation of the accuracy of continuous glucose sensors. J Diabetes Sci Technol, $2,853-62$.

CAPES, S. E., HUNT, D., MALMBERG, K. \& GERSTEIN, H. C. 2000. Stress hyperglycaemia and increased risk of death after myocardial infarction in patients with and without diabetes: a systematic overview. Lancet, 355, 773-778.

CARAYON, P. \& GURSES, A. P. 2005. A human factors engineering conceptual framework of nursing workload and patient safety in intensive care units. Intensive Crit Care Nurs, 21, 284-301.

CHASE, J. G., ANDREASSEN, S., JENSEN, K. \& SHAW, G. M. 2008. Impact of Human Factors on Clinical Protocol Performance: A Proposed Assessment Framework and Case Examples. Journal of Diabetes Science and Technology, 2, 409-416.

CHEE, F., FERNANDO, T. \& VAN HEERDEN, P. V. 2003a. Closed-loop glucose control in critically ill patients using continuous glucose monitoring system (CGMS) in real time. IEEE Trans Inf Technol Biomed, 7, 43-53.

CHEE, F., FERNANDO, T. L., SAVKIN, A. V. \& VAN HEEDEN, V. 2003b. Expert PID control system for blood glucose control in critically ill patients. IEEE Trans Inf Technol Biomed, 7, 419-25.

CLARKE, W. L., ANDERSON, S., FARHY, L., BRETON, M., GONDER-FREDERICK, L., COX, D. \& KOVATCHEV, B. 2005. Evaluating the clinical accuracy of two continuous glucose sensors using continuous glucose-error grid analysis. Diabetes Care, 28, 2412-2417.

EGI, M., BELLOMO, R., STACHOWSKI, E., FRENCH, C. J. \& HART, G. 2006. Variability of blood glucose concentration and short-term mortality in critically ill patients. Anesthesiology, 105, 244-52.

EGI, M., BELLOMO, R., STACHOWSKI, E., FRENCH, C. J., HART, G. K., TAORI, G., HEGARTY, C. \& BAILEY, M. 2010. Hypoglycemia and outcome in critically ill patients. Mayo Clin Proc, 85, 217-24.

FINNEY, S. J., ZEKVELD, C., ELIA, A. \& EVANS, T. W. 2003. Glucose control and mortality in critically ill patients. Jama, 290, 2041-2047.

GOLDBERG, P. A., SIEGEL, M. D., RUSSELL, R. R., SHERWIN, R. S., HALICKMAN, J. I., COOPER, D. A., DZIURA, J. D. \& INZUCCHI, S. E. 2004. Experience with the continuous glucose monitoring system in a medical intensive care unit. Diabetes Technol Ther, 6, 339-47.
HARRIS, D. L., BATTIN, M. R., WESTON, P. J. \& HARDING, J. E. 2010. Continuous Glucose Monitoring in Newborn Babies at Risk of Hypoglycemia. Journal of Pediatrics, 157, 198-202.

HERMANIDES, J., BOSMAN, R. J., VRIESENDORP, T. M., DOTSCH, R., ROSENDAAL, F. R., ZANDSTRA, D. F., HOEKSTRA, J. B. \& DEVRIES, J. H. 2010. Hypoglycemia is associated with intensive care unit mortality. Crit Care Med, 38, 1430-4.

KLONOFF, D. C. 2005a. Continuous Glucose Monitoring: Roadmap for 21 st century diabetes therapy. Diabetes Care, 28, 1231-9.

KLONOFF, D. C. 2005b. A review of continuous glucose monitoring technology. Diabetes Technol Ther, 7, $770-5$.

KRINSLEY, J. S. 2003. Association between hyperglycemia and increased hospital mortality in a heterogeneous population of critically ill patients. Mayo Clin Proc, $78,1471-1478$

KRINSLEY, J. S. 2008. Glycemic variability: a strong independent predictor of mortality in critically ill patients. Crit Care Med, 36, 3008-13.

LIN, J., LEE, D., CHASE, J. G., SHAW, G. M., LE COMPTE, A., LOTZ, T., WONG, J., LONERGAN, T. \& HANN, C. E. 2008. Stochastic modelling of insulin sensitivity and adaptive glycemic control for critical care. Computer Methods and Programs in Biomedicine, 89, 141-152.

MACKENZIE, I., INGLE, S., ZAIDI, S. \& BUCZASKI, S. 2005. Tight glycaemic control: a survey of intensive care practice in large English hospitals. Intensive Care Med, 31, 1136.

SKYLER, J. S. 2009. Continuous glucose monitoring: an overview of its development. Diabetes Technol Ther, 11 Suppl 1, S5-10.

VAN DEN BERGHE, G., WOUTERS, P., WEEKERS, F., VERWAEST, C., BRUYNINCKX, F., SCHETZ, M., VLASSELAERS, D., FERDINANDE, P., LAUWERS, P. \& BOUILLON, R. 2001. Intensive insulin therapy in the critically ill patients. $N$ Engl $J$ Med, 345, 1359-1367. 